\title{
Diurnal variation of Martian water-ice clouds in Tharsis region of the low latitude cloud belt: Observations in 1995-1999 apparitions
}

\author{
T. Akabane ${ }^{1}$, T. Nakakushi ${ }^{2}$, K. Iwasaki ${ }^{3}$, and S. M. Larson ${ }^{4}$ \\ 1 Hida Observatory, Kyoto University, Kamitakara, Gifu 506-1314, Japan \\ 2 Kwasan Observatory, Kyoto University, Yamashina, Kyoto 607-8471, Japan \\ e-mail: kushio@kusastro.kyoto-u.ac.jp \\ 3 Kyoto Gakuen University, Sogabecho, Kameoka, Kyoto 621-8555, Japan \\ e-mail: iwasaki@kyotogakuen.ac.jp \\ 4 Lunar and Planetary Laboratory, University of Arizona, Tucson, Arizona 85721, USA \\ e-mail: slarson@pirlmail.lpl.arizona.edu
}

Received 3 April 2001 / Accepted 7 December 2001

\begin{abstract}
We investigated diurnal variations of water-ice clouds in the Tharsis region of Mars in the three apparitions from 1995 to 1999 . The Tharsis morning cloud, centered near a point $\left(120^{\circ} \mathrm{W}, 10^{\circ} \mathrm{N}\right)$ between Olympus Mons and Tharsis Montes, was the brightest among the morning clouds in Tharsis. Its optical thickness was about 0.6 near $9.5 \mathrm{~h}$ Martian local time and reduced to a minimum of about 0.2 around local noon in 1995. The minimum optical thickness was 0.4 in 1997, which also appeared around local noon. The optical thickness of the Tharsis morning cloud increased again in the early or mid afternoon in 1995 and 1997. In the late morning when the brightness of the morning cloud declined, bright afternoon clouds appeared over the major volcanoes in Tharsis. The optical thickness of the afternoon cloud over Olympus Mons was about 0.7 in the early afternoon in early summer, 1997. The Olympus afternoon cloud seems to be more active in early summer than in the first half of late spring. Those morning and afternoon clouds repeat the diurnal variation from late spring to at least early summer every Martian year.
\end{abstract}

Key words. planets and satellites: individual: Mars - planets and satellites: general - atmospheric effects

\section{Introduction}

A Martian cloud can have one of two forms: volatile (or white) clouds and dust (or yellow) clouds. Some volatile clouds may consist of $\mathrm{CO}_{2}$ ice particles, but most of them in low to mid latitudes in northern summer may be water-ice clouds. Hereafter the term "cloud" refers to a water-ice cloud. The investigation of Martian clouds is important for the study of Martian climate. Since the Viking era, the Martian climate has been simulated by global circulation models (e.g., Pollack et al. 1990). Seasonally appearing Martian clouds may be one constraint for the circulation models. Morphological features and optical characteristics of Martian clouds have been secured by several techniques (Table 1). Slipher (1962) and Martin et al. (1992) summarize the morphological features of clouds observed by ground-based telescopes.

Send offprint requests to: T. Akabane,

e-mail: akabane@kwasan.kyoto-u.ac.jp
Owing to those and other observations it is known that three types of cloud appear in low latitudes of both hemispheres and mid latitudes of the northern hemisphere in northern spring to summer. They are morning, evening, and afternoon clouds. Morning and evening clouds extend frequently from southern low latitudes to northern mid latitudes, covering wide areas. Afternoon clouds are visible as bright spots in the afternoon, but are much smaller than morning and evening clouds. They appear on large volcanoes such as Elysium Mons and Olympus Mons in the northern hemisphere. Afternoon clouds begin to be visible in early spring of the northern hemisphere. First they are visible only near the evening limb or terminator, and their brightness increases as the season advances towards late spring.

In spring to summer of the northern hemisphere, clouds in low latitudes form a seasonal cloud belt (Clancy et al. 1996). The cloud belt is called the "equatorial cloud belt" (James et al. 1996), "low latitude cloud belt" (Clancy et al. 1996), or "aphelion cloud belt" 
Table 1. Cloud observations (some examples).

\begin{tabular}{ll}
\hline Visual observations & Miyamoto (1965, 1968, 1974) \\
$\begin{array}{l}\text { Imaging observations } \\
\text { Earth based }\end{array}$ & Slipher (1962), Martin \& Baum (1969); \\
& Smith \& Smith (1972); Martin \& McKinney (1974); \\
& Hattori \& Akabane (1974); Akabane et al. (1980, 1987, 1995); \\
& Parker et al. (1999) \\
Mariner 9 & Leovy et al. (1972, 1973); Curran et al. (1973); Briggs \& Leovy (1974) \\
Viking 1, 2 & Briggs et al. (1977); Piekersgill \& Hunt (1981); French et al. (1981); Christensen \& Zurek (1984) \\
HST $^{*}$ & James et al. (1994, 1996); Wolff et al. (1999) \\
Polarimetric observations & Dollfus (1961); Dollfus et al. (1989, 1996); Lee et al. (1990) \\
\hline
\end{tabular}

* Hubble Space Telescope.

(Wolff et al. 1999). In this paper we use the term "low latitude cloud belt". The most active period of the cloud belt is late spring to early summer and the cloud belt disappears by early autumn (Wolff et al. 1999; Tamppari et al. 2000). Most of the morning, evening, and afternoon clouds lie in the cloud belt. Regions in which all of the three types of cloud appear are restricted to two large orogenic areas in northern low latitudes: Elysium and Tharsis. The afternoon clouds on Elysium Mons (Elysium afternoon cloud) is as bright as those on Olympus Mons (Olympus afternoon cloud), but morning and evening clouds in Elysium are much less active than those in Tharsis. Clouds in Tharsis are bright and conspicuous, and have been morphologically studied. The classical "W cloud" is characteristic of Tharsis (Slipher 1962). The morning, evening, and afternoon clouds in Tharsis repeat the diurnal variation for a long period in northern spring to summer (Slipher 1962; Smith \& Smith 1972; Briggs et al. 1977). The regular appearance of clouds in the cycle of a day may suggest that meteorological conditions repeat in the same period. However, quantitative investigations of the diurnal variation of the cloud activity are few. We quantitatively show the cloud activity in recent apparitions and compare it to our earlier observations.

The vertical optical thickness may be one of criterions of cloud activity. But optical thicknesses of Martian clouds have not yet been observed directly from the Martian ground. Curran et al. (1973) derived the optical thickness of clouds over Tharsis to be about 0.4 in early summer from the Mariner 9 data in 1972. It was the average over the field of view of the Mariner 9 instrument. They suggest that the optical thickness of the brightest region of the cloud may be four times larger. From the Viking data at $L \mathrm{~s}=29^{\circ}$ in 1979 ( $L \mathrm{~s}$ is the areocentric orbital longitude of the Sun), Christensen \& Zurek (1984) estimated the optical thickness of a cloud over Olympus Mons to be of the order of unity or larger. Narumi et al. (1987) derived the optical thickness of a morning cloud at $90^{\circ} \mathrm{W}$ longitude on the equator around $L \mathrm{~s}=116^{\circ}$ from imaging observations in 1982. The optical thickness was about 3 near $7 \mathrm{~h}$ MLT (Martian local time) and decreased to about 0.2 around local noon. The cloud repeated almost the same diurnal variation during their observations from April 18 to 25, 1982. Clancy et al. (1996) derived optical thicknesses of clouds in low latitudes from the Hubble Space Telescope (HST) observations. The optical thickness of a cloud at $120^{\circ} \mathrm{W}$ and $10^{\circ} \mathrm{N}$ in Tharsis was 0.7 in the mid morning at $L \mathrm{~s}=94^{\circ}$ in 1993. Analyzing the HST images, Wolff et al. (1999) derived the optical thickness of a morning cloud at $33{ }^{\circ} \mathrm{W}$ and $0^{\circ}-30{ }^{\circ} \mathrm{N}$ in Chryse at $L \mathrm{~s}=98^{\circ}$ (1997). It was 0.3 at $10.5 \mathrm{~h}$ MLT. Wolff et al. (1999) also showed seasonal variations of cloud opacities in the low latitude cloud belt near Syrtis Major, in Amazonis, and in Chryse. Their Figs. 7-9 suggest that the cloud belt had not yet existed in the Syrtis Major region at $L \mathrm{~s}=40^{\circ}$, and that it was visible at $L \mathrm{~s}=65^{\circ}$, although the cloud belt appeared in the Amazonis region at $L \mathrm{~s}=45^{\circ}$.

In this paper we investigate the diurnal variation of the optical thickness of morning clouds centered at $120^{\circ} \mathrm{W}$ and $10^{\circ} \mathrm{N}$ in Tharsis and afternoon clouds on Olympus Mons in the 1995 to 1999 apparitions. It was mid spring to early summer in the northern hemisphere. We represent the transient features from morning to afternoon clouds in Tharsis in Sect. 3, and diurnal variations of morning clouds over Tharsis and of afternoon clouds over Olympus Mons are represented in Sect. 4. The distribution of the optical thickness of clouds on a parallel of $10{ }^{\circ} \mathrm{N}$ in the low latitude cloud belt is shown in Sect. 5 .

\section{Observations and reductions}

We made CCD imaging observations in 1995 to 1999 apparitions of Mars in order to investigate cloud activity in mid spring to early summer of the northern hemisphere of Mars. In those seasons water vapor is most abundant in the northern hemisphere (Jakosky \& Farmer 1982), and many bright clouds are visible not only in the northern hemisphere but also over large basins in the southern mid latitudes. We used the $65 \mathrm{~cm}$ refractor at the Hida Observatory, Japan, for our observations. Around the opposition of Mars in April, 1999, we had the opportunity to use the $1.52 \mathrm{~m}$ reflector and the $1.55 \mathrm{~m}$ reflector at the Catalina Observatory near Tucson, Arizona. Ephemerides for the relevant observations are given in Table 2 .

In this investigation, we reduced images exposed through blue filters, characteristics of which are summarized in Table 3 . For the calibration of data points on each image, we selected a reference point near the central 
Table 2. Ephemerides for relevant observations.

\begin{tabular}{llrllrl}
\hline & $\begin{array}{l}\text { Date } \\
\text { (UT) }\end{array}$ & $\begin{array}{r}L_{\mathrm{S}} \\
(\mathrm{deg})\end{array}$ & $\begin{array}{l}D_{\mathrm{S}} \\
(\mathrm{deg})\end{array}$ & $\begin{array}{l}D_{\mathrm{E}} \\
(\mathrm{deg})\end{array}$ & $\alpha$ & Station \\
\hline 1995 & & & & & & \\
& Mar. 6 & 68 & +23 & +17 & 17 & Hida \\
& Mar. 7 & 68 & +23 & +17 & 18 & Hida \\
& Mar. 28 & 77 & +25 & +17 & 29 & Florida* \\
& Mar. 31 & 78 & +25 & +17 & 30 & Florida* \\
& Apr. 13 & 84 & +25 & +18 & 34 & Hida \\
1997 & & & & & & \\
& Feb. 14 & 78 & +25 & +23 & -23 & Hida \\
& Mar. 5 & 86 & +25 & +23 & -10 & Hida \\
& Mar. 24 & 95 & +25 & +23 & 6 & Hida \\
& Apr. 1 & 98 & +25 & +24 & 13 & Hida \\
& Apr. 26 & 110 & +24 & +25 & 28 & Hida \\
& & & & & & \\
& Mar. 16 & 110 & +24 & +15 & -27 & Hida \\
& Apr. 20 & 127 & +20 & +18 & -4 & Mt. Lemmon \\
& Apr. 27 & 130 & +19 & +19 & 2 & Mt. Bigelow \\
\hline
\end{tabular}

$L_{\mathrm{S}}$ : Areocentric orbital longitude of the Sun.

$D_{\mathrm{S}}$ : Latitude of sub-solar point.

$D_{\mathrm{E}}$ : Latitude of sub-Earth point.

$\alpha$ : Phase angle. Minus sign $(-)$ means pre-opposition.

*: D. Parker's observations.

Table 3. Telescopes and filters.

\begin{tabular}{lcccccr}
\hline \multirow{2}{*}{ Station } & \multicolumn{2}{c}{ Telescope } & \multicolumn{2}{c}{ CCD } & \multicolumn{2}{c}{ Filter } \\
\cline { 2 - 7 } & $\begin{array}{c}D \\
(\mathrm{~cm})\end{array}$ & $\begin{array}{c}f \\
(\mathrm{~m})\end{array}$ & $\begin{array}{c}\text { Pixel size } \\
(\mu \mathrm{m})\end{array}$ & $\begin{array}{c}\text { Resolution } \\
(\operatorname{arcsec} / \mathrm{pix})\end{array}$ & $\begin{array}{c}\lambda_{0} \\
(\mathrm{~nm})\end{array}$ & $\begin{array}{r}\Delta \lambda_{1 / 2} \\
(\mathrm{~nm})\end{array}$ \\
\hline Hida Obs. & 65 & 20.7 & 9 & 0.09 & 436.2 & 15.6 \\
Mt. Lemmon & 152 & 28.0 & 24 & 0.18 & 426.6 & 6.4 \\
Mt. Bigelow & 155 & 20.9 & 14 & 0.14 & 426.6 & 6.4 \\
\hline
\end{tabular}

$D$ : Diameter.

$f$ : Equivalent focal length.

$\lambda_{0}$ : Wavelength at peak transparency.

$\Delta \lambda_{1 / 2}$ : Full width at half maximum.

meridian in mid latitudes of the northern hemisphere where it seems to be free from cloud. The intensity at a data point on a cloud or at the reference point was defined as the mean of the intensities of $3 \times 3$ pixels at the center of which was the data or reference point. We selected the best three among 6 to 20 images exposed within a few minutes. The relative intensity of the data point to the reference point was obtained for each of the best three images, and the average of the three relative intensities was adopted for the relative brightness of the data point to the reference point.

The relative brightness of the data point was used to derive the optical thickness of cloud at the data point with the assumption that the reference point was free from cloud. The uncertainty, which is the standard deviation divided by the mean, was within $4 \%$ in the relative brightness of data points. Larger values of uncertainties in the relative brightness in data points occurred near the limb. We selected data points at which incident and emergent angles were less than $70^{\circ}$. The uncertainty was within $5 \%$ of the optical thickness for most of the data points.

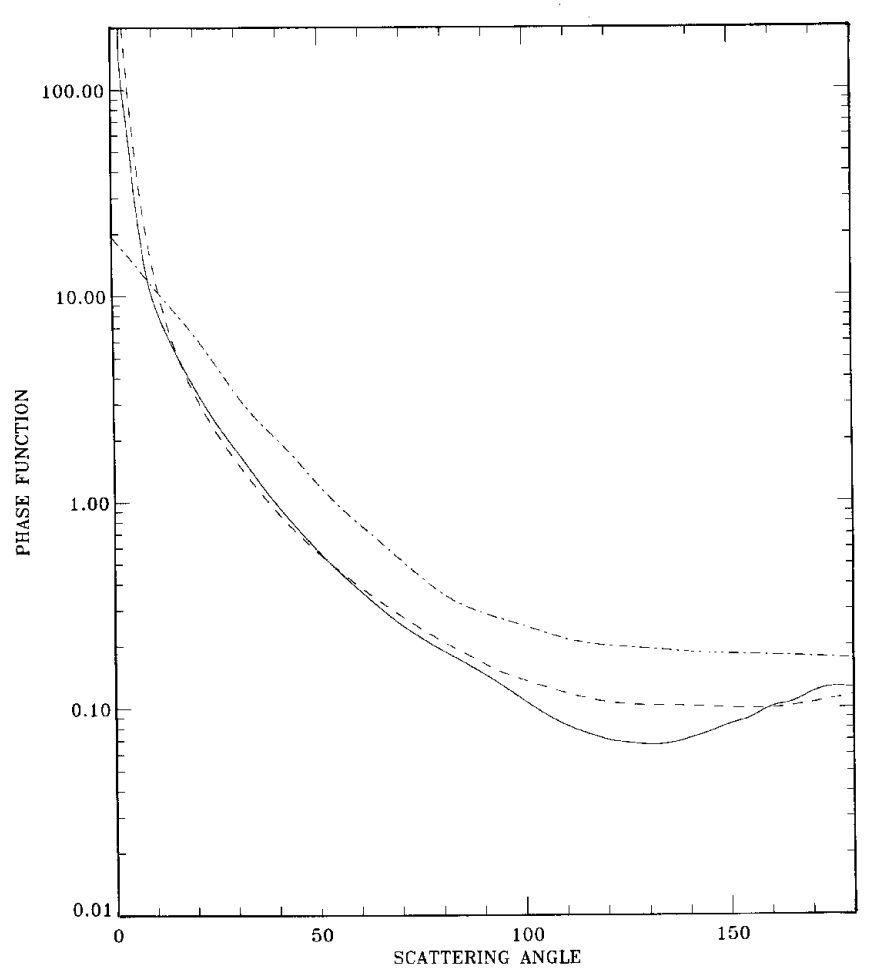

Fig. 1. Scattering phase functions used in this investigation. Solid line represents the phase function for water-ice particles measured in the laboratory (Sassen \& Liou 1979), and dashed line for Martian dust observed by Pathfinder (Tomasko et al. 1999). Clancy \& Lee's (1991) phase function is shown (dash dot line), which is derived from Martian clouds in mid latitudes by Viking observations.

The optical thickness was derived from the equation of radiative transfer. The discrete ordinate method was used to solve the equation (Chandrasekhar 1950). The algorithm for the computer code was from Liou (1973), Wiscombe (1977), Stamnes \& Swanson (1981), Stamnes \& Dale (1981), and Stamnes et al. (1988). We assumed a uniform atmosphere of $6.1 \mathrm{mb}$ containing dust equivalent to the opacity of 0.3 in our observational period (Clancy et al. 2000; Toigo \& Richardson 2000). The scattering phase function for Martian dust in blue was calculated with parameters in Table 8 of Tomasco et al. (1999). We adopted Sassen \& Liou's (1979) scattering phase function for Martian clouds (Fig. 1). It was measured at the wavelength of $640 \mathrm{~nm}$ in a laboratory for ice particles of mean size $3.5 \mu \mathrm{m}$.

The Minnaert function (Minnaert 1941) was used to calculate the reflectance at the ground.

$R=R_{0} \mu_{0}^{k} \mu^{k-1}$

where $\mu_{0}$ and $\mu$ are cosines of incident and scattering angles, respectively. The coefficient $R_{0}$ depends on the wavelength $(\lambda)$ and phase angle $(\alpha)$ (e.g. Kieffer et al. 1977). We estimated $R_{0}$ from parameters for Flats soil observed by Mars Pathfinder (Johnson et al. 1999). For example, it is $0.059,0.051$, and 0.040 , respectively, at $\alpha=2^{\circ}, 10^{\circ}$, and $30^{\circ}$. The Minnaert exponent $k$ depends 
also on $\lambda$ and $\alpha$. We adopted $k=0.5$ at small phase angles $\left(\alpha<10^{\circ}\right)$ and $k=0.6$ for larger ones $\left(\alpha \geq 10^{\circ}\right)$ in blue. The adopted values of $R_{0}$ and $k$ are briefly discussed in Sect. 6 .

\section{Morphological features of morning and afternoon clouds in Tharsis}

Clouds over the Tharsis region are very active. Bright afternoon clouds are conspicuous over huge volcanoes such as Olympus Mons. Morning and evening clouds are also bright.

There is a particularly bright morning cloud between Olympus Mons and the three volcanoes of Tharsis Montes. Its center is about $120^{\circ} \mathrm{W}$ and $10^{\circ} \mathrm{N}$. We call this bright spot the "Tharsis morning cloud". A good example of the morning cloud was observed by the HST at $L \mathrm{~s}=94^{\circ}$ in 1993 as seen in Fig. 16 of Clancy et al. (1996).

The Tharsis morning cloud disappears or its brightness reduces extremely in the early afternoon. As the morning cloud dissipates, small bright spots of clouds begin to appear over the large volcanoes. Images on the upper row in Fig. 2 show the sequence of the event observed on March 6 , 1995. Martian local time near the center of the morning cloud $\left(120^{\circ} \mathrm{W}, 10^{\circ} \mathrm{N}\right)$ was $9.64 \mathrm{~h}, 10.36 \mathrm{~h}$, and $11.41 \mathrm{~h}$, respectively, in images $\mathrm{a}, \mathrm{b}$, and $\mathrm{c}$. The morning cloud in image a was the brightest and largest among the three images. The morning cloud in image b declined in its brightness and dimensions, and in image c, exposed one hour later than image b, the Tharsis morning cloud can hardly be identified. Its brightness reduced to the same level as neighboring clouds, but small and bright spots appeared on the borders of the morning cloud. The location of the eastern spot corresponds to Ascraeus Mons and the western spot to Olympus Mons. Those bright spots are the afternoon clouds on volcanoes. Their brightness increased one hour later (image d). On the next day, March 7, the same transient phenomenon from the morning cloud to afternoon clouds was seen (lower row in Fig. 2). The Tharsis morning cloud was bright at 9.46 h MLT in image e. But the brightness reduced much during one hour, and afternoon clouds appeared as bright spots (image f). The local time at the appearance of morning cloud was $10.72 \mathrm{~h}$ (image $\mathrm{f}$ ). The transient features suggest that the morning cloud declined around $11 \mathrm{~h}$ MLT and afternoon clouds became visible over volcanoes.

About 5 weeks later, the Tharsis region faced us again and the Tharsis morning cloud was visible. Figure 3 shows the morning clouds on April 13, $1995\left(L \mathrm{~s}=84^{\circ}\right)$. The local time at the point $\left(120^{\circ} \mathrm{W}, 10^{\circ} \mathrm{N}\right)$ was $8.66 \mathrm{~h}, 9.27 \mathrm{~h}$, and $10.14 \mathrm{~h}$, respectively, in images from left to right. Olympus Mons, Acraeus Mons, and Pavonis Mons are visible as dark spots. These dark spots surrounded by white clouds suggest that the altitude of the Tharsis morning cloud is not high compared to the top of the volcanoes. The HST and microwave observations show that summits of the volcanoes were visible above the cloud and that the water vapor in low to mid latitudes was confined to altitudes below
$10 \mathrm{~km}$ in the 1993 and 1995 aphelion periods (Clancy et al. 1996). On the contrary the large volcanoes are not identified in images exposed on March 6 and 7, 1995 (See Fig. 2). On those days $\left(L \mathrm{~s}=67^{\circ}\right)$ the altitude of the cloud might have been higher than the volcanoes.

Afternoon clouds tend to appear over large volcanoes such as Olympus Mons. Afternoon clouds in the Tharsis region are already visible by early spring. The HST observed afternoon clouds in the Tharsis region at $L \mathrm{~s}=11^{\circ}$ in 1996 (James \& Lee 1996, Home page for HST Mars, Photo No. PRC96-34.) and at $L_{\mathrm{s}}=20^{\circ}$ in 1993 (James et al. 1996). We observed them at $L \mathrm{~s}=35^{\circ}$ in 1994 at Hida. However they are not as bright as those in late spring to early summer. Similar to morning clouds, afternoon clouds repeat the regular diurnal variation. In the most active period, afternoon clouds begin to be visible in the mid morning. For example, the Olympus afternoon cloud appeared before $10 \mathrm{~h}$ MLT in 1995, when the Tharsis morning cloud declined in brightness. We can identify the Olympus afternoon cloud in Figs. 2c and $\mathrm{f}$, in which the local time at Olympus Mons was $9.98 \mathrm{~h}$ and $9.29 \mathrm{~h}$, respectively. Afternoon clouds may increase their brightness as they approach the evening limb, as did the Olympus afternoon cloud in 1982 (Akabane et al. 1987). However, afternoon clouds merge into evening clouds (Figs. 2d, h). Sometimes afternoon clouds form the "W cloud" (Slipher 1962) together with evening clouds. Bright spots in the $\mathrm{W}$-cloud may be afternoon clouds.

\section{Optical thicknesses of the Tharsis morning cloud and the Olympus afternoon cloud}

The Tharsis morning cloud and the Olympus afternoon cloud are brighter and more conspicuous than other clouds in the Tharsis region. The optical thickness of the cloud is one of criteria of the cloud activity. In order to estimate the diurnal variation of the activity of the Tharsis morning cloud and the Olympus afternoon cloud, optical thicknesses were derived. The data point for the Tharsis morning cloud was taken at a point $120{ }^{\circ} \mathrm{W}$ and $10^{\circ} \mathrm{N}$ which was near the center of the cloud, and that for the Olympus afternoon cloud was taken at the cloud center.

Figure 4 shows the diurnal variation of the optical thickness of the Tharsis morning cloud observed in 1995. The optical thickness was about 0.5 near $9.5 \mathrm{~h}$ MLT on March 6 and $7\left(L \mathrm{~s}=68^{\circ}\right)$. It decreased to about 0.15 near local noon. On April $13\left(\mathrm{Ls}=84^{\circ}\right)$ the optical thickness was about 1.1 at $9.3 \mathrm{~h}$ MLT, corresponding to twice the optical thickness than in the March 6 and 7 cases. Parker (1995) got images exposed through a blue filter (Schott BG12+IR rejection) on March $28\left(L \mathrm{~s}=77^{\circ}\right)$ and 31, 1995. The optical thicknesses of the Tharsis morning cloud on those days are also included in Fig. 4. The optical thickness was about 0.9 near $8 \mathrm{~h}$ MLT on March 31 and was about 0.6 near 9.5 h MLT on March 28 and 31, 1995. The optical thicknesses near $9.5 \mathrm{~h}$ MLT in 1995 were about $0.5,0.6$, and 1.1, respectively, at $L \mathrm{~s}=68^{\circ}$ (early March), $L \mathrm{~s}=77^{\circ}$ (late March), and $L \mathrm{~s}=84^{\circ}$ (mid April), that is, 

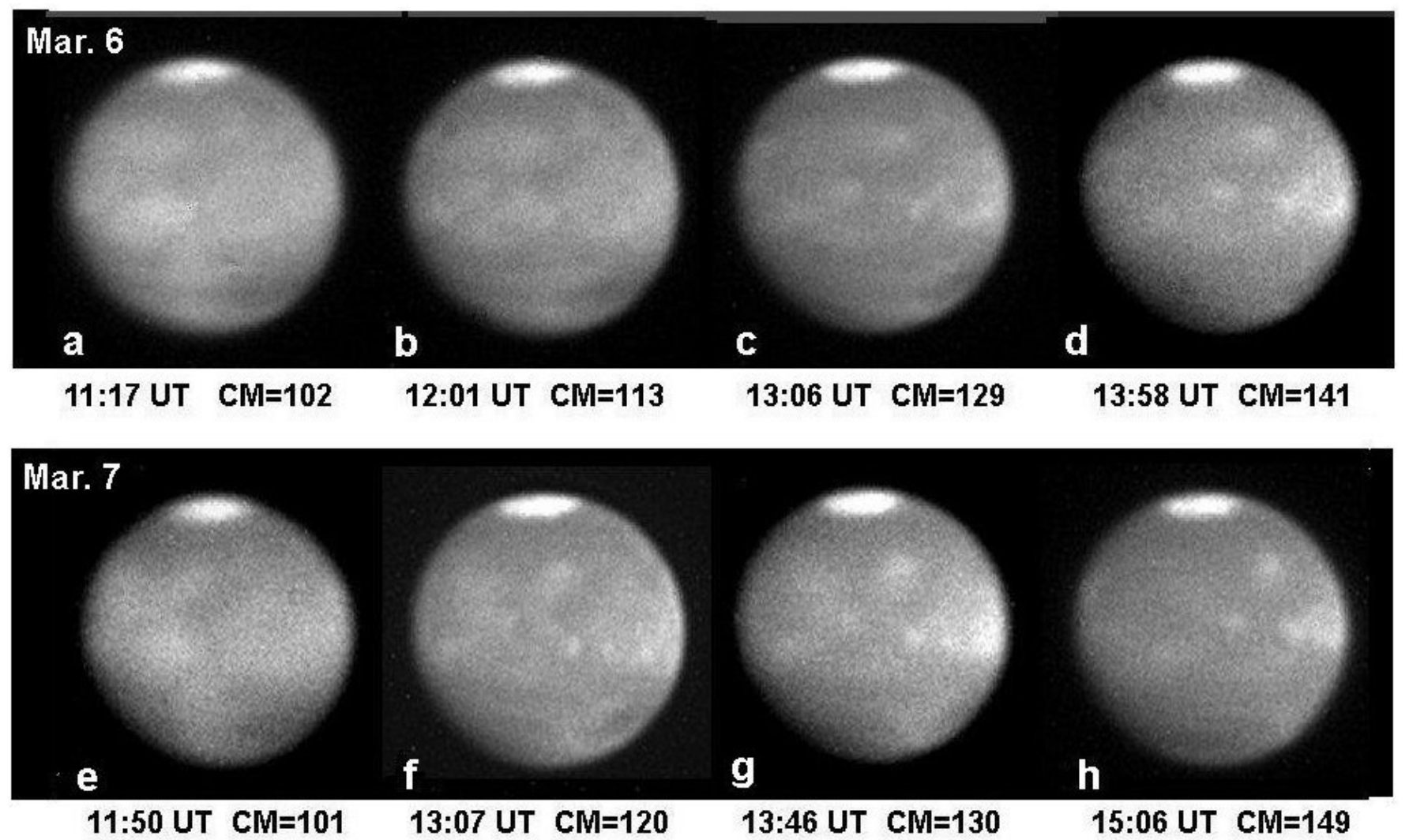

Fig. 2. Tharsis morning cloud and afternoon clouds on March 6 (upper row) and March 7 (lower row) in $1995\left(L \mathrm{~s}=68^{\circ}\right)$. The brightness of the morning cloud reduces in the late morning, and then afternoon clouds appear on large volcanoes. On March 6, the Tharsis morning cloud is visible in images a) and b), but invisible in image c) where afternoon clouds appear on Olympus Mons (near the disk center) and Ascraeus Mons. On March 7, the Tharsis morning cloud is invisible in image f), but an afternoon cloud is visible on Ascraeus Mons. Olympus afternoon cloud is visible in images $\mathbf{g}$ ) and $\mathbf{h}$ ). The local time near the center of the cloud $\left(120^{\circ} \mathrm{W}, 10^{\circ} \mathrm{N}\right)$ in images a)-d) on March 6 is, respectively, $9.64 \mathrm{~h}, 10.36 \mathrm{~h}, 11.41 \mathrm{~h}$, and $12.25 \mathrm{~h}$, and that on March 7 is $9.53 \mathrm{~h}, 10.79 \mathrm{~h}, 11.42 \mathrm{~h}$, and $12.72 \mathrm{~h}$, respectively, in images $\mathbf{e})-\mathbf{h})$.

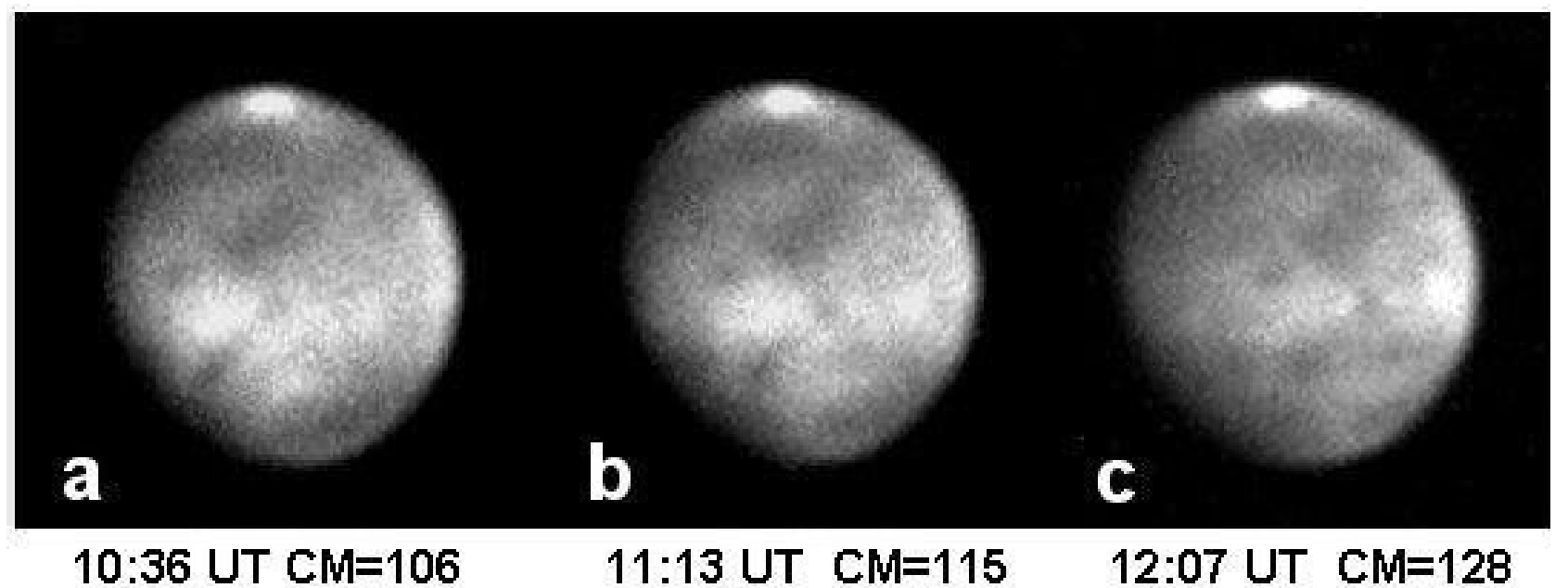

Fig. 3. Tharsis morning cloud on April 13, $1995\left(\mathrm{Ls}=94^{\circ}\right)$. The local time at a point $\left(120^{\circ} \mathrm{W}, 10^{\circ} \mathrm{N}\right.$; about the cloud center $)$ is $8.66 \mathrm{~h}, 9.27 \mathrm{~h}$, and $10.14 \mathrm{~h}$, respectively, in images a)-c).

the optical thickness seems to have increased with season from $L \mathrm{~s}=68^{\circ}$ to $84^{\circ}$ in 1995 .

Narumi et al. (1987) measured the relative brightness of morning clouds at $90^{\circ} \mathrm{W}$ on the equator (Cloud I) and at $120^{\circ} \mathrm{W}$ and $10^{\circ} \mathrm{N}$ (Cloud II) in images exposed in
1982 at Hida, and estimated the optical thicknesses of the clouds. Cloud II is the Tharsis morning cloud cited in this paper. However, optical thicknesses of Cloud II (Tharsis morning cloud) are not presented in their paper. We recalculated those optical thicknesses using phase functions 


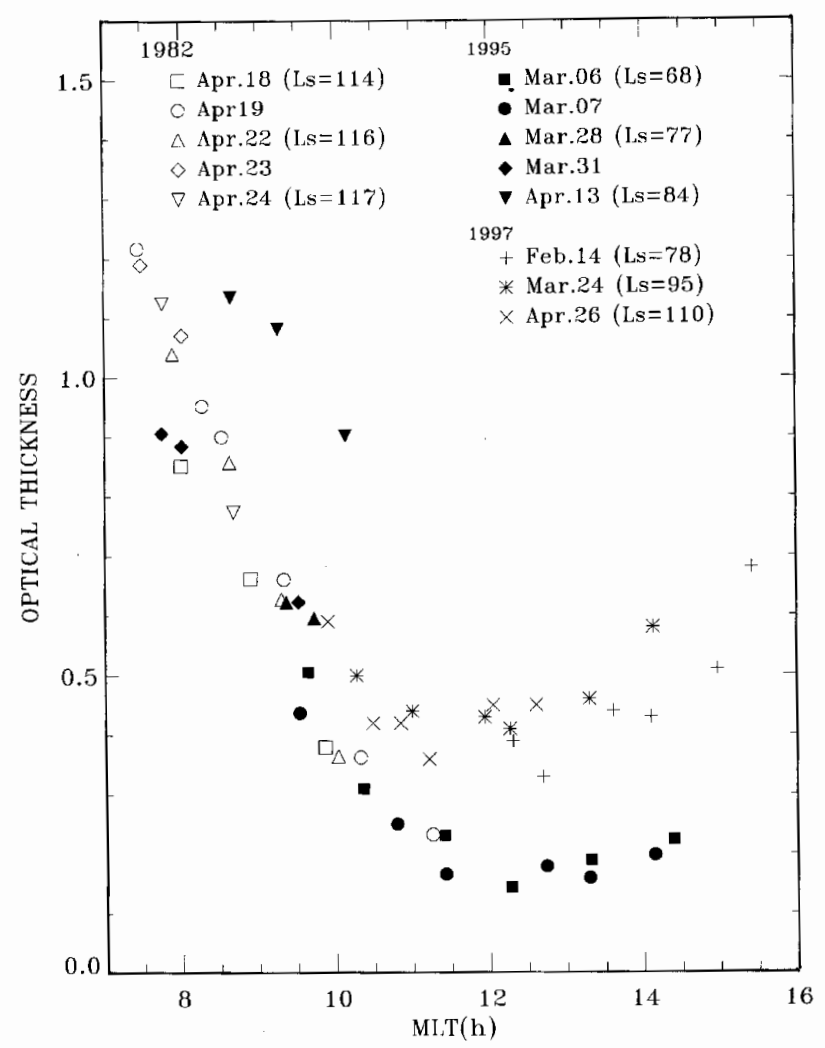

Fig. 4. Diurnal variations of the optical thickness of the Tharsis morning cloud in $1995\left(L \mathrm{~s}=68^{\circ}-84^{\circ}\right)$ and 1997 $(L \mathrm{~s}=78-110)$. Also those in $1982\left(L \mathrm{~s}=114^{\circ}-117^{\circ}\right)$ are shown for comparison. The optical thickness of the morning cloud reaches a minimum around noon and then increases in the mid afternoon.

and the ground reflectivity described in Sect. 2. They are represented in Fig. 4 to compare with those in 1995. The optical thicknesses in the early morning are somewhat higher in 1982 than in 1995, but there is no significant difference between them.

In 1995 and 1997, we could follow the Tharsis morning cloud from the late morning to the early afternoon. In 1997 the afternoon cloud over Ascraeus Mons was large and extended westward near the point $\left(120^{\circ} \mathrm{W}, 10^{\circ} \mathrm{N}\right)$ in the early afternoon, so that we selected the data point at $\left(130^{\circ} \mathrm{W}, 10^{\circ} \mathrm{N}\right)$ for the cloud in 1997 in place of $\left(120^{\circ} \mathrm{W}\right.$, $\left.10^{\circ} \mathrm{N}\right)$. The optical thickness decreased to a minimum around local noon, and then increased again with the local time (Fig. 4). Nakakushi et al. (2001) derived the diurnal variation of cloud opacities over Arabia, Syrtis Major, and Isidis from observations in $1997\left(L \mathrm{~s}=87^{\circ}\right)$ and 1999 $\left(L \mathrm{~s}=127^{\circ}-129^{\circ}\right)$. Those morning clouds decreased their optical thickness with time during the morning as did the Tharsis morning cloud, but did not disappear, keeping minimum optical thicknesses in the early afternoon. The behavior of the morning clouds in Tharsis and ArabiaIsidis suggests that morning clouds in low latitudes are visible even in the afternoon in a period from late spring to

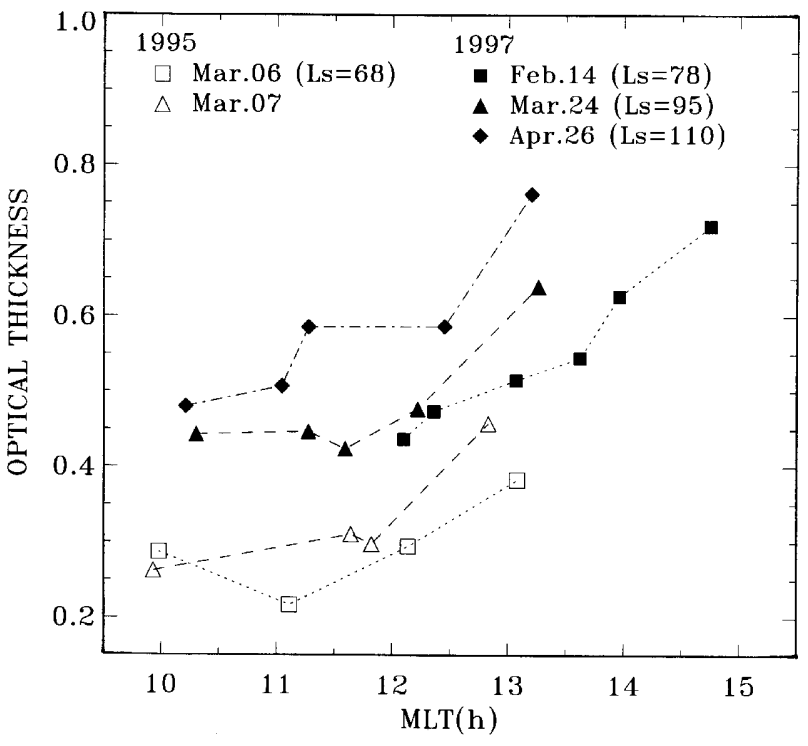

Fig. 5. Optical thickness of Olympus afternoon cloud in 1995 $\left(L \mathrm{~s}=68^{\circ}\right)$ and $1997\left(L \mathrm{~s}=78^{\circ}-110^{\circ}\right)$. The optical thickness is higher in 1997 than in 1995. It suggests that the afternoon clouds become more active as the season advances from the first half of late spring to early summer.

early summer. The optical thickness of the Tharsis morning cloud in the late morning to early afternoon in 1997 $\left(L \mathrm{~s}=78^{\circ}-109^{\circ}\right)$ is higher than that in $1995\left(L \mathrm{~s}=68^{\circ}\right)$. This may show that the Tharsis morning cloud is more active around the summer solstice than in the first half of late spring.

The Olympus afternoon cloud is the brightest among the afternoon clouds in the Tharsis region. It was already visible around $10 \mathrm{~h}$ MLT in $1995\left(L \mathrm{~s}=68^{\circ}\right)$. Due to the high optical thickness of morning clouds, the Olympus cloud was not identified before 9 h MLT. Figure 5 shows the diurnal variation of the optical thickness of the Olympus cloud in 1995 and 1997. The brightness and the optical thickness of the Olympus cloud increased with the local time after 11h MLT. In our observations in 1995 and 1997 we could not confirm when the brightness and the optical thickness reached their peaks in the diurnal variation. According to observations in 1982, the brightness of the Olympus cloud increased as it approached the evening limb due to the limb effect increasing the pathlength in the cloud (Akabane et al. 1987). The optical thickness of the Olympus cloud in 1982 reached a peak between $14 \mathrm{~h}$ and $15 \mathrm{~h}$ MLT. The peak opacity was about 0.4 . However, it must be underestimated. In Akabane et al.'s (1987) paper, the mean of the brightness on east and west borders of the cloud was adopted as the reference brightness for the calibration of the brightness of the Olympus afternoon cloud. However, the Olympus afternoon cloud in 1982 lay in the low latitude cloud belt whose opacity might have been about 0.2 even in the early afternoon, as discussed in the next section. Therefore the optical thickness of the Olympus afternoon cloud in 1982 should be increased by about 0.2 . Then the peak value of the optical 
thickness in 1982 should be about 0.6, which is consistent with that in the early afternoon in 1997. The optical thickness of the Olympus afternoon cloud in 1997 is higher than that in 1995. This may be due to the progression of the season from $L \mathrm{~s}=68^{\circ}$ (1995) to $L \mathrm{~s}=110^{\circ}$ (1997). The Olympus afternoon cloud repeated such a diurnal variation in that the optical thickness increased from the late morning to the early afternoon, similar to the case in 1982.

\section{Morning-to-evening side variation of the optical thickness in the low latitude cloud belt}

According to the HST observations, the low latitude cloud belt becomes visible between $L \mathrm{~s}=40^{\circ}$ and $60^{\circ}$ as seen in Fig. 1 of James et al. (1994) and Figs. 7-9 of Wolff et al. (1999). Erard (2000) suggests that the formation stage of the cloud belt is $L \mathrm{~s}=50^{\circ}$ to $60^{\circ}$. Although it has not been precisely confirmed yet when the cloud belt disappears, its most active stage may be late spring to early summer in the northern hemisphere (Tamppari et al. 2000).

The low latitude cloud belt consists of morning, evening, and afternoon clouds. The width of the belt extends from $10^{\circ} \mathrm{S}$ to $30^{\circ} \mathrm{N}$ in the active stage (Clancy et al. 1996; Wolff et al. 1999). Figure 6 shows the low latitude cloud belt in the Arabia, Amazonis, and Chryse regions in 1997 and 1999. The morning and evening features of Tharsis are seen in the Chryse and Amazonis regions, respectively. Near the limb or terminator the cloud belt is bright and often extends to higher latitudes due to developed morning and evening clouds. The cloud belt reduces in width and brightness in the late morning to mid afternoon areas. Also the brightness in the cloud belt is different from area to area. Clouds in the Amazonis region are not conspicuous in an area from $140^{\circ} \mathrm{W}$ to $210^{\circ} \mathrm{W}$ compared to those in the Tharsis and Arabia regions.

Wolff et al. (1999) estimated optical thicknesses of clouds in the low latitude cloud belt. Their Fig. 8 shows that the peak optical thickness appears in late spring to early summer. Also the same figure suggests that the cloud belt disappears around $L_{\mathrm{s}}=135^{\circ}$ (mid summer of the northern hemisphere), although morning clouds are still visible.

We estimated the optical thickness of the low latitude cloud belt from the morning to evening sides along a latitude line of $10^{\circ} \mathrm{N}$ in the cloud belt. Figures 7 and 8 show optical thicknesses of the cloud belt in the Arabia, Amazonis, and Chryse regions on March $5\left(L \mathrm{~s}=86^{\circ}\right)$, $24\left(L \mathrm{~s}=95^{\circ}\right)$, and April $1\left(L \mathrm{~s}=98^{\circ}\right)$ in 1997, and March $16\left(L \mathrm{~s}=110^{\circ}\right)$, April $20\left(L \mathrm{~s}=127^{\circ}\right)$ and $27(L \mathrm{~s}=$ $130^{\circ}$ ) in 1999 , respectively. Note that Figs. 7 and 8 do not show a diurnal variation of the optical thickness of a cloud at the same point, but show a variation of the optical thickness from the morning to evening sides in the images in Fig. 6. The optical thickness of the morning part in the Chryse region is much higher than those in the other regions. The morning part corresponds to morning clouds over Tharsis. In the Chryse region the optical thickness decreases to its minimum near $14 \mathrm{~h}$ MLT. It is about $0.2 \mathrm{in}$
1997, while about 0.5 in 1999 . Toigo \& Richardson (2000) reanalyzed Viking observations, and derived the optical thickness of clouds in the afternoon at the Viking Lander I site (Chryse) assuming the dust opacity ratio of visible to infrared to be 2.5. Their optical thicknesses range from 0.1 to 0.5 in northern spring to summer, and the mean is about 0.3 around $L \mathrm{~s}=90^{\circ}$. Our optical thicknesses of the low latitude cloud belt in the afternoon part are consistent with those of Toigo \& Richardson (2000).

In the Arabia and Amazonis regions, optical thicknesses were about 0.2 around noon. Optical thicknesses in the mid afternoon part in the Amazonis region are higher than those in other regions. That part corresponds to the area where the Tharsis morning cloud appears. The optical thickness in late morning to mid afternoon parts in the Chryse region in 1999 was much higher than that in 1997. Also, the optical thickness near Syrtis Major around noon in 1995 was about 3 times as high as that in 1993 (Wolff et al. 1999). The optical thickness of the cloud belt may vary significantly annually in certain areas. The optical thickness curves in Figs. 7 and 8 differ from one another. It may be due to local effects such as topography, temperature, and amount of water vapor in the atmosphere.

The features of the diurnal variations of morning clouds over Tharsis (Fig. 4) are similar to those of the low latitude cloud belt in Figs. 7 and 8. The optical thickness of the low latitude cloud belt in the midday area was about 0.2 . The Tharsis morning cloud did not disappear even at midday, and the minimum optical thickness was about 0.2 in 1995 . In late spring to early summer, which may be the most active period of clouds in low latitudes, most morning clouds in low latitudes may not disappear even in the early or mid afternoon when evening clouds begin to grow. Most clouds in the late morning to the early afternoon parts of the cloud belt may be morning clouds that are reduced in brightness and optical thickness but still are visible and recover their brightness in the mid to late afternoon as evening clouds. Evening clouds which form the evening part of the cloud belt may be the recondensation of vapor from the morning clouds.

\section{Discussions and conclusions}

The Minnaert coefficient $R_{0}$ in Eq. (1) is a product of a normal albedo at the incident angle of $0(A n)$ and a phase function of the ground $(\Phi(\alpha))$ (e.g., Kieffer et al. 1977).

$R_{0}=A n \Phi(\alpha)$.

Referring to Eqs. (10.28) and (10.32) of Hapke (1993), An and $\Phi(\alpha)$ are

$$
\begin{aligned}
& A n=\frac{\omega_{0}}{8}\left(1+B_{0}\right) p(0) \\
& \Phi(\alpha)=\frac{1+B(\alpha)}{1+B_{0}} \frac{p(\alpha)}{p(0)} \\
& B(\alpha)=\frac{B_{0}}{1+\frac{1}{h} \tan (\alpha / 2)}
\end{aligned}
$$




\section{Arabia Region \\ Amazonis Region \\ Chryse Region}
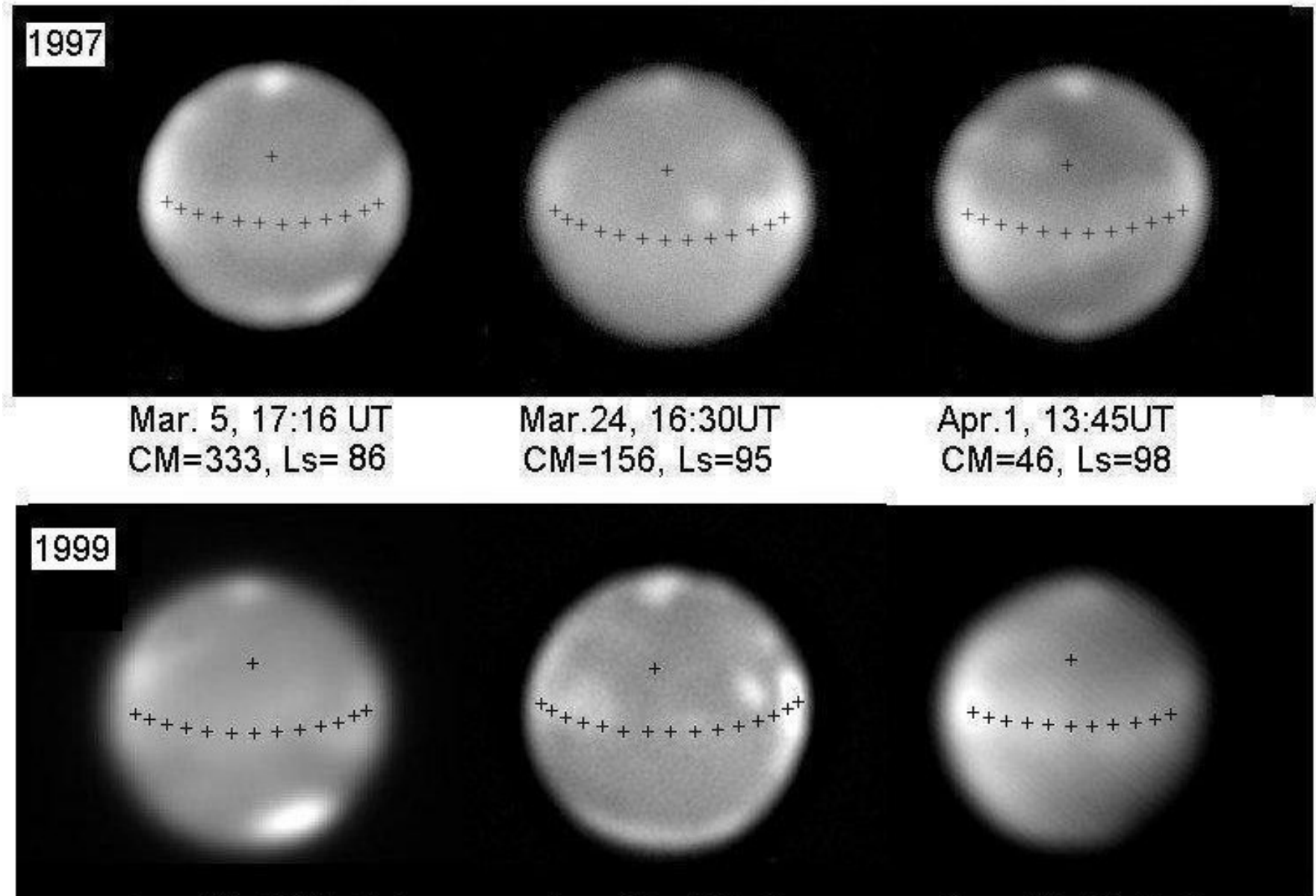
Apr. 20, 10:04 UT
Apr. 27, 4:33 UT
Mar. 16, 19:49 UT
$\mathrm{CM}=323$, Ls $=127$
$\mathrm{CM}=181, \mathrm{Ls}=130$
$\mathrm{CM}=58$, Ls=111

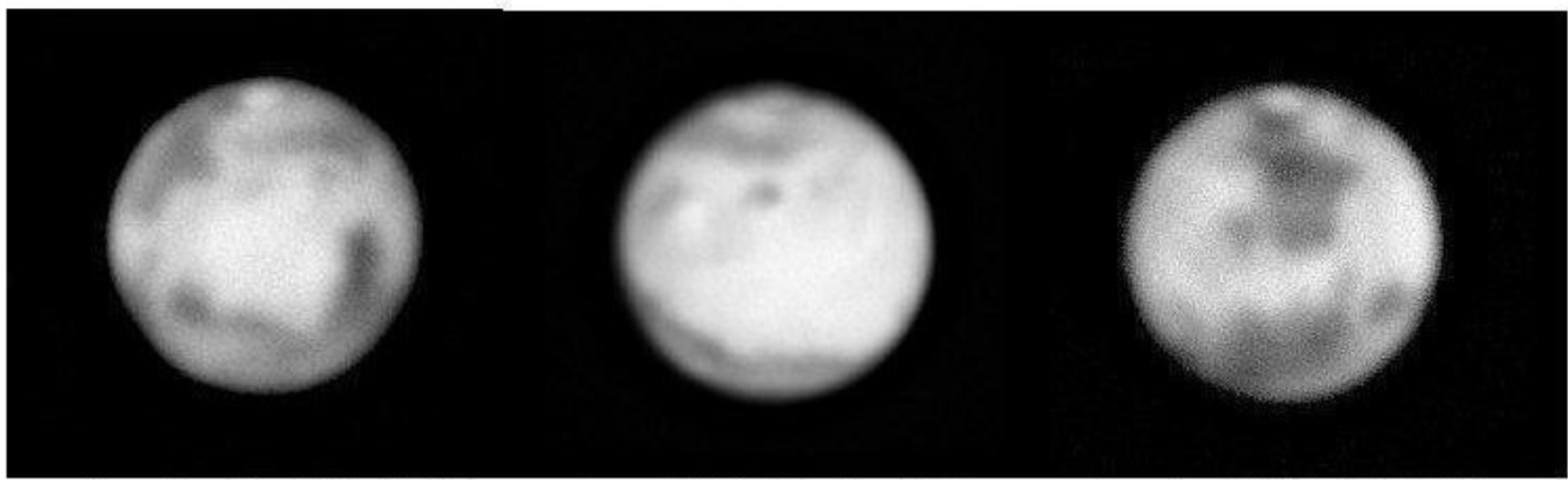
Mar. 5, 17:01 UT('97)
Apr. 27, 4:21 UT ('99)
Apr. 1, 13:33 UT ('97)
$\mathrm{CM}=329, \mathrm{Ls}=86$
$\mathrm{CM}=178, \mathrm{Ls}=130$
$\mathrm{CM}=41, \mathrm{Ls}=98$

Fig. 6. Low latitude cloud belt in the Arabia, Amazonis and Chryse regions in 1997 and 1999. Crosses in the cloud belt lie on the $10{ }^{\circ} \mathrm{N}$ latitude, and most of them (i.e., except ones near the limb) correspond to data points at which optical thicknesses are estimated in Figs. 7 and 8. Crosses in mid latitudes are reference points for the calibration of intensities at data points. Albedo features in those regions are seen on images in the lowest row. 


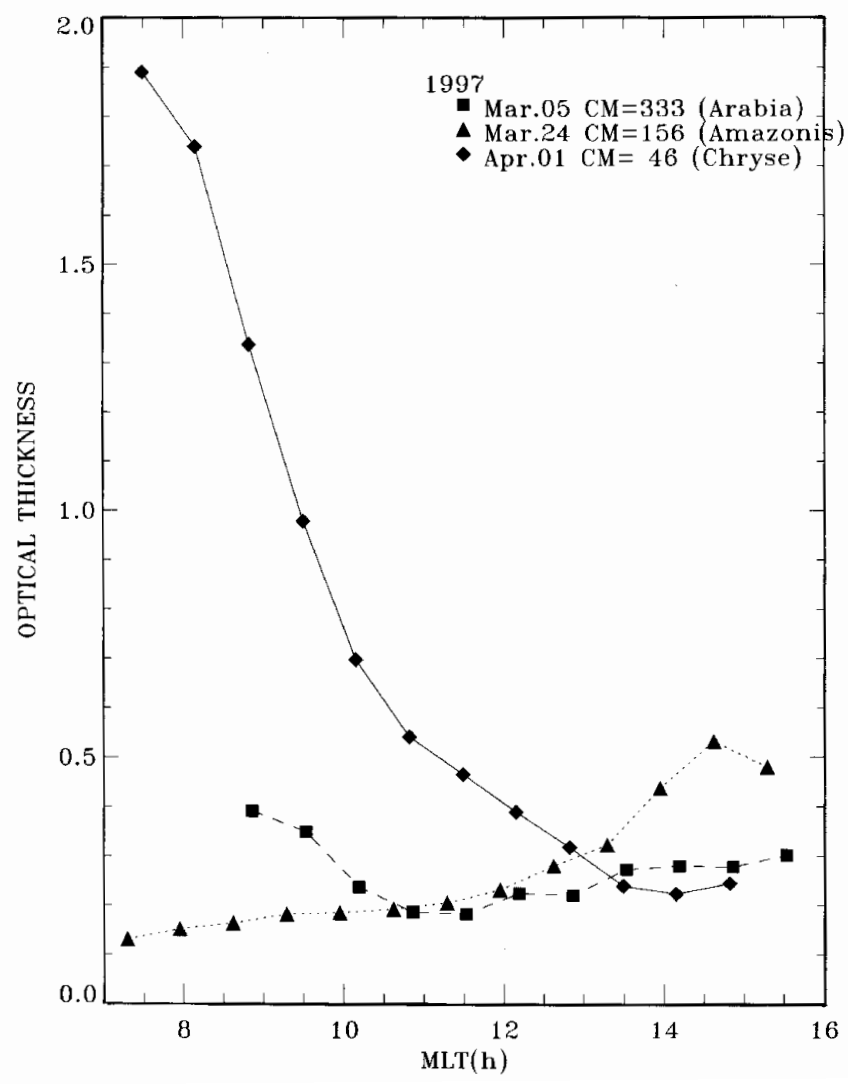

Fig. 7. Distribution of the optical thickness in the low latitude cloud belt along a parallel of $10{ }^{\circ} \mathrm{N}$ in 1997 in the Arabia, Amazonis, and Chryse regions. The optical thicknesses in the early morning part are much higher in the Chryse region than the other regions. The higher optical thicknesses correspond to morning clouds in Tharsis.

at each phase angle we adopted parameters derived from Flats soil at the Pathfinder site (Johnson et al. 1999).

The Minnaert exponent $k$ depends also on the wavelength and phase angle (e.g., Veverka \& Wasserman 1972; Veverka et al. 1989; McEven 1991). We adopted $k=0.5$ for small phase angles and $k=0.6$ for larger phase angles. The earth-based observations show no center-to-limb variation in the brightness on the Martian disk in blue at the phase angle of $5^{\circ}$ (Lumme et al. 1981). In the Martian atmosphere loaded with dust equivalent to the opacity of 0.3 , the uniform brightness of the disk in blue at $\alpha=5^{\circ}$ can be reproduced with $k=0.5$. At large phase angles the center-to-limb variation in the brightness cannot be reproduced with $k=0.5$. We examined the brightness variation in low latitudes of the northern hemisphere on an image exposed on November 3, $1992\left(L \mathrm{~s}=350^{\circ}, \alpha=38^{\circ}\right)$. It was a cloud-free season. We found that the best fit value of $k$ was 0.6. However we could not find the phase angle at which the best fit value of $k$ shifts from 0.5 to 0.6. Referring to Table 4 of Erard (2000) we adopted $k=0.5$ for $\alpha<10^{\circ}$ and $k=0.6$ for $\alpha \geq 10^{\circ}$ in blue.

The optical thicknesses of clouds derived in the above sections depend largely on the scattering phase function of

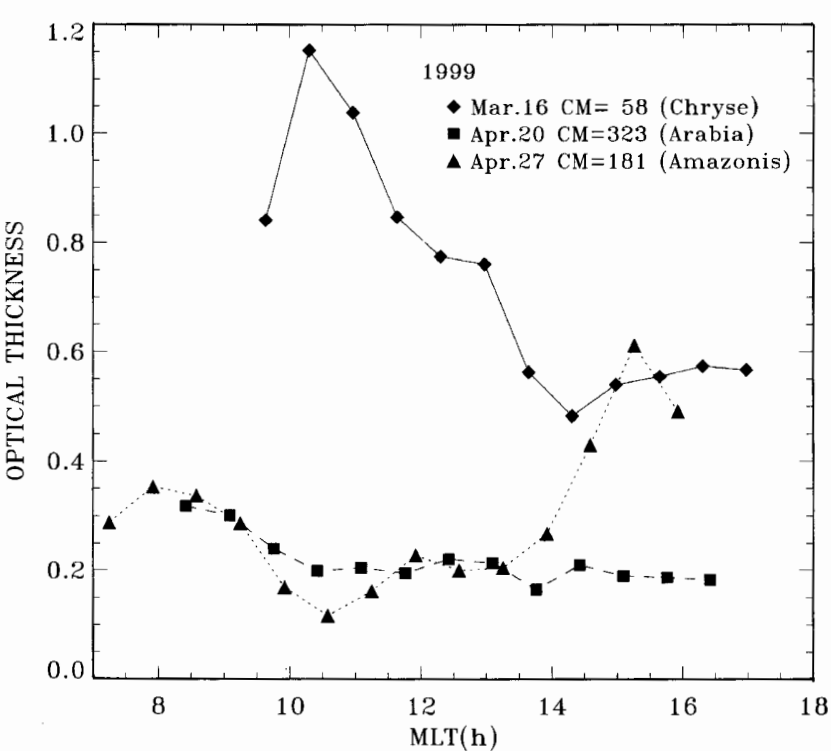

Fig. 8. Same as Fig. 7, but in 1999.

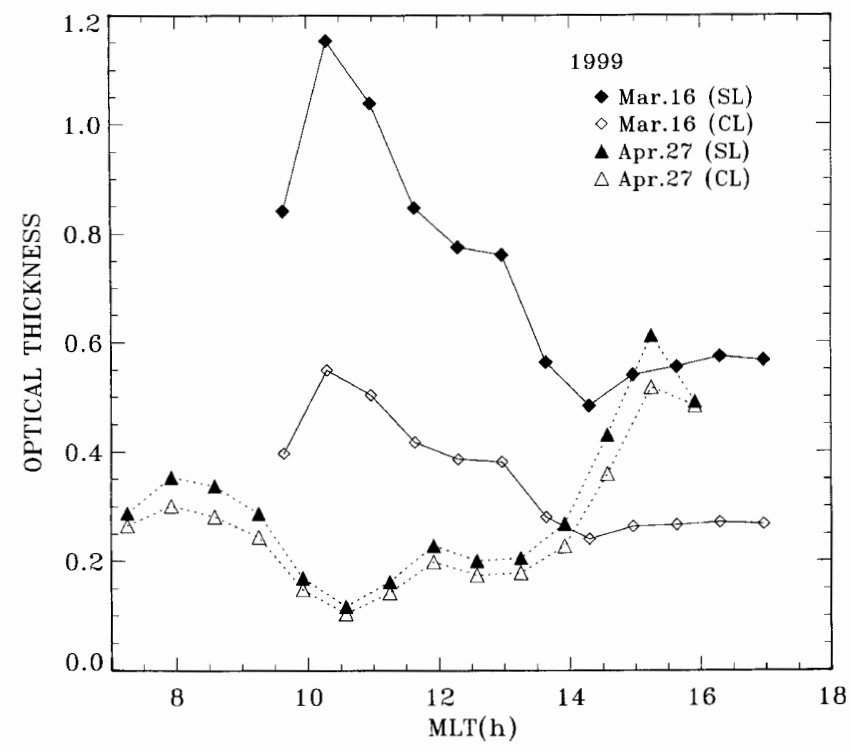

Fig. 9. Comparison of optical thicknesses derived from the Sassen \& Liou's (1979) phase function (filled symbols/SL) to those from the Clancy \& Lee's (1991) phase function (open symbols/CL). The difference between optical thicknesses from the two is large on March 16 when the phase angle was $27^{\circ}$, and is small on April 27 when it was $2^{\circ}$.

cloud. We adopted Sassen \& Liou's (1979) phase function (SL phase function hereafter). Clancy \& Lee (1991) derived a phase function of Martian cloud in mid latitudes from the Viking Orbiters' observations (CL phase function hereafter). The range of the phase angle where they measured the phase function directly was about $\alpha=35^{\circ}$ to $\alpha=135^{\circ}$. The CL phase function is nearly constant at 
small phase angle, and higher than the SL phase function except at large phase angle (Fig. 1). In our observational period, the phase angle was within $30^{\circ}$. In this range the difference between the SL and CL phase functions increases with the phase angle, i.e., the SL phase function is about 0.7 times the CL phase function at $\alpha=0^{\circ}$ and about 0.4 times at $\alpha=30^{\circ}$. Due to these differences, optical thicknesses derived from the SL phase function are much higher than those from the CL phase function near $\alpha=30^{\circ}$. Figure 9 shows the difference in the case of the low latitude cloud belt on April $27\left(\alpha=2^{\circ}\right)$ and March $16\left(\alpha=27^{\circ}\right), 1999$. The optical thicknesses derived from the SL phase function are about twice those from the CL phase function at $\alpha=27^{\circ}$, and the difference is small at the small phase angle $\left(\alpha=2^{\circ}\right)$.

The optical thicknesses of clouds may depend also on the opacity of dust in the atmosphere of Mars. The recent investigations show that the dust opacity is about 0.2-0.4 under the normal atmospheric conditions in late spring to early summer of the northern hemisphere (James et al. 1994; Wolff et al. 1999; Clancy et al. 2000; Toigo \& Richardson 2000). However, according to opacity observations of the Martian sky by Viking Landers (Pollack et al. 1979; Colburn et al. 1989) and Pathfinder (Smith \& Lemmon 1999), dust in the atmosphere is about 0.5 in opacity in the northern summer period. We recalculated the optical thickness of the low latitude cloud belt on April 27, 1999 for two cases of dust opacity: 0.5 and 0.2 . The result is that the cloud optical thickness increased about $10 \%$ on morning and evening sides and decreased about $10 \%$ around local noon in the case of dust opacity 0.5 compared to the case of dust opacity 0.2 . Thus the dust opacity of 0.5 or 0.2 does not significantly affect the cloud optical thickness described in the above sections.

We assumed a constant atmospheric pressure of $6.1 \mathrm{mb}$. The pressure varies topographically and seasonally. Even if it varies from $2 \mathrm{mb}$ to $10 \mathrm{mb}$, the opacity of the $\mathrm{CO}_{2}$ atmosphere is on order of 0.01 in blue, which is negligibly small compared to the dust opacity of 0.3 . Therefore the fluctuations in the atmospheric pressure due to the topography and season do not affect the estimation of the optical thickness of clouds.

In summary, the optical thickness of the Tharsis morning cloud was more than 1 in the early morning and reduced to about 0.2 around noon in 1995. A similar behavior of the Tharsis morning cloud was observed in 1982. The Tharsis morning cloud in 1995 and 1997 did not disappear, even in the early afternoon, keeping a minimum optical thickness and then recovering optical thickness in the mid afternoon. The Olympus afternoon cloud appeared in the late morning and increased in brightness with local time in 1995 and 1997. The optical thickness was about 0.7 in the early afternoon in 1997, consistent with that in 1982 . Observations in 1982 and 1995-1997 suggest that morning and afternoon clouds almost regularly repeat their activities every Martian year.

The distribution of the optical thickness along a latitude of $10^{\circ} \mathrm{N}$ shows that the optical thickness tends to be higher in the mid morning and evening parts of the cloud belt than in the midday part where it is about 0.2 . The optical thickness in the midday part is consistent with the minimum of the Tharsis morning cloud, suggesting that the low latitude cloud belt appears when morning clouds do not disappear, even in the afternoon.

We presented diurnal variations of the Tharsis morning cloud and Olympus afternoon cloud. They may be representatives of morning and afternoon clouds, respectively, in low latitudes in northern spring to summer. Although they differ in growth period and/or process, both Tharsis morning cloud and Olympus afternoon cloud repeat diurnal variations regularly for a long period at least from late spring to early summer. These findings should be considered in studies of Martian meteorology.

Acknowledgements. We are grateful to Donald C. Parker for furnishing images of Mars secured in Florida for this study. We thank Ann Sprague and Sumisaburo Saito for their critical reading of the manuscript and helpful suggestions. Also we thank an anonymous referee for his/her useful comments.

\section{References}

Akabane, T., Matsui, M., Ishiura, K., et al. 1980, Contri. Kwasan Hida Obs., Univ. Kyoto, No. 242

Akabane, T., Iwasaki, K., Saito, Y., \& Narumi, Y. 1987, Pub. Astron. Soc. Jpn., 39, 343

Akabane, T., Saito, Y., Iwasaki, K., \& Martin, L. J. 1995, A\&A, 304, 595

Briggs, G. A., Klaasen, K., Thorpe, T., Wellman, J., \& Baum, W. 1977, J. Geophys. Res., 82, 4121

Briggs, G. A., \& Leovy, C. B. 1974, Bull. Am. Meteorol. Sos., 55,278

Chandrasekhar, S. 1950, Radiative transfer (Oxford Univ. Press, London)

Christensen, P. R., \& Zurek, R. W. 1984, J. Geophys. Res., 89, 4587

Clancy, R. T., \& Lee, S. W. 1991, Icarus, 93, 135

Clancy, R. T., Grossman, A. W, Wolff, M. J., et al. 1996, Icarus, 122,36

Clancy, R. T., Sandor, B. J., Wolff, M. J., et al. 2000, J. Geophys. Res., 105, 9553

Colburn, D. S., Pollack, J. B., \& Haberle, R. M. 1989, Icarus, 79,159

Curran, R. J., Conrath, B. J., Hanel, R. A., Kunde, V. G., \& Pearl, J. C. 1973, Science, 182, 381

Dollfus, A. 1961, Polarization study of planets, in Planets and satellites, ed. G. P. Kuiper, \& B. M. Middlehurst (Univ. Chicago Press, Chicago), 343

Dollfus, A., Ebisawa, S., \& Bowell, E. 1984, A\&A, 131, 123

Dollfus, A., Ebisawa, S., \& Crussaire, D. 1996, J. Geophys. Res., 101, 9207

Erard, S. 2000, Planet. Space Sci., 48, 1271

French, R. G., Gierasch, P. J., Popp, B. O., \& Yerdon, R. J. 1981, Icarus, 45, 468

Hapke, B. 1993, Theory of reflectance and emittance spectroscopy (Cambridge Univ. Press, Cambridge) 
Hattori, A., \& Akabane, T. 1974, Contr. Inst. Astrophys. Kwasan Obs, Univ. Kyoto, No. 221

Jakosky, B. M., \& Farmer, C. B. 1982, J. Geophys. Res., 87, 2999

James, P. B., Clancy, R. T., Lee, S. W., et al. 1994, Icarus, 109, 79

James, P. B., Bell III, J. F., Clancy, R. T., et al. 1996, J. Geophys. Res., 101, 18883

Johnson, J. R., Kirk, R., Soderblom, L. A., et al. 1999. J. Geophys. Res., 104, 8809

Kieffer, H. H., Martin, T. Z., Peterfreund, A. R., et al. 1977, J. Geophys. Res., 82, 4249

Lee, P., Ebisawa, S., \& Dollfus, A. 1990, A\&A, 240, 520

Leovy, C. B., Briggs, G. A., Young, A. T., et al. 1972, Icarus, 17,373

Leovy, C. B., Briggs, G. A., \& Smith, B. A. 1973, J. Geophys. Res., 78, 4252

Liou, K.-N. 1973, J. Atmos. Sci., 30, 1303

Lumme, K., Martin, L. J., \& Baum, W. A. 1981, Icarus, 45, 379

Martin, L. J., \& Baum, W. A. 1969, A study of cloud motions on Mars, Final report, Part B. Planetary Research Center, Lowell Observatory, Flagstaff, Arizona, USA

Martin, L. J., \& Mckinney, W. M. 1974, Icarus, 23, 380

Martin, L. J., James, P. B., Dollfus, A., Iwasaki, K., \& Beish, J. D. 1992, Telescopic observations: Visual, photographic, polarimetric. in Mars, ed. H. H. Kieffer, B. M. Jakosky, C. W. Snyder, \& M. S. Matthews (Univ. Arizona Press, Tucson), 34

McEven, A. S. 1991, Icarus, 92, 298

Minnaert, M. 1941, ApJ, 93, 403

Miyamoto, S. 1965, Contr. Inst. Astrophys. Kwasan Obs., Univ. Kyoto, No. 141

Miyamoto, S. 1968, Contr. Inst. Astrophys. Kwasan Obs., Univ. Kyoto, No. 169

Miyamoto, S. 1974, Contr. Kwasan Hida Obs., Univ. Kyoto, No. 217
Narumi, Y., Akabane, T., Iwasaki, K., \& Saito, Y. 1987, Proceedings of the 20th ISAS Lunar and Planetary Symposium (Institute of Space and Astronautical Science, Tokyo), 7

Nakakushi, T., Akabane, T., Iwasaki, K., \& Larson, S. M. 2001, J. Geophys. Res., 106, 5043

Parker, D. C. 1995, private communication

Parker, D. C., Beish, J. D., Troiani, D. M., Joyce, D. P., \& Hernandez, C. E. 1999, Icarus, 138, 3

Pickersgill, A. O., \& Hunt, G. E. 1981, J. Atmos. Sci., 38, 40

Pollack, J. B., Colburn, D. S., Flasar, F. M., Kahn, R., Carlston, C. E., \& Pidek, D. 1979, J. Geophys. Res., 84, 2929

Pollack, J. B., Haberle, R. M., Schaeffer, J. \& Lee, H. 1990, J. Geophys. Res., 95, 1447

Sassen, K., \& Liou, K.-N. 1979, J. Atmos. Sci., 36, 838

Slipher, E. C. 1962, The photographic story of Mars (Northland Press, Flagstaff, Arizona)

Smith, P. H., \& Lemmon, M. 1999, J. Geophys. Res., 104, 8975

Smith, S. A., \& Smith, B. A. 1972, Icarus, 16, 509

Stamnes, K., \& Dale, H. 1981, J. Atmos. Sci., 38, 2696

Stamnes, K., \& Swanson, R. A. 1981, J. Atmos. Sci., 38, 387

Stamnes, K., Tsay, S-C., Wiscombe, W., \& Jayaweera, K. 1988, App. Opt., 27, 2502

Tamppari, L. K., Zurek, R. Z., \& Paige, D. A. 2000, J. Geophys. Res., 105, 4087

Toigo, A. D., \& Richardson, M. I. 2000, J. Geophys. Res., 105, 4109

Tomasko, M. G., Doose, L. R., Lemmon, M., Smith, P. H., \& Wegryn, E. 1999, J. Geophys. Res., 104, 8987

Veverka, J., \& Wasserman, L. 1972, Icarus, 16, 281

Veverka, J., Helfenstein, P., Skypeck, A., \& Thomas, P. 1989, Icarus, 78,14

Wiscombe, W. J. 1977, J. Atmos. Sci., 34, 1408

Wolff, M. J., Bell III, J. F., James, P. B., Clancy, R. T., \& Lee, S. W. 1999, J. Geophys. Res., 104, 9027 